\title{
Serum N-terminal telopeptide of type I collagen as an early marker of fracture nonunion in rabbits
}

\author{
JIAN-PING LIN ${ }^{1,2}$, ZHAN-JUN SHI $^{1}$, NING-JIANG SHEN ${ }^{2}$, JIAN WANG $^{1}$, ZAO-MIN LI $^{2}$ and JUN XIAO ${ }^{1}$ \\ ${ }^{1}$ Department of Orthopedic Surgery, Nanfang Hospital, Southern Medical University, Guangzhou, Guangdong 510515; \\ ${ }^{2}$ Department of Orthopedic Surgery, Hainan Provincial People's Hospital, Haikou, Hainan 570311, P.R. China
}

Received January 30, 2015; Accepted March 8, 2016

DOI: $10.3892 / \mathrm{etm} .2016 .3839$

\begin{abstract}
The aim of the present study was to establish an experimental animal model of fracture nonunion, and to investigate the changes in serum biomarker concentrations in fracture nonunion. A total of 20 purebred New Zealand rabbits were divided into two group: A bone defect group and a bone fracture group. In the bone defect group, a $15-\mathrm{mm}$ section of bone (including the periosteum) was removed from the mid-radius, and the medullary cavities were closed with bone wax. In the bone fracture group, the mid-radius was fractured. $\mathrm{X}$-rays were taken and blood samples were collected preoperatively and at 2, 3, 4, 5, 6, 7, 8, 10 and 12 weeks after the surgical procedure. The serum concentrations of osteocalcin (OC) and bone-specific alkaline phosphatase (BSAP) served as markers of bone formation, and those of C-terminal telopeptide of type I collagen (CTX), N-terminal telopeptide of type I collagen (NTX) and tartrate-resistant acid phosphatase 5b (TRACP 5b) served as markers of bone resorption. The concentration levels of the markers were measured using a biotin double-antibody sandwich enzyme-linked immunosorbent assay. In the bone defect group, bone callus was observed on X-ray at 2 weeks in three rabbits and the bone calluses stabilized at 5 weeks;
\end{abstract}

Correspondence to: Dr Zhan-Jun Shi, Department of Orthopedic Surgery, Nanfang Hospital, Southern Medical University, 1023 ShaTai South Road, Guangzhou, Guangdong 510515, P.R. China

E-mail: shizhanjun2014@163.com

Dr Jian-Ping Lin, Department of Orthopedic Surgery, Hainan Provincial People's Hospital, 19 Xiuhua Road, Haikou, Hainan 570311, P.R. China

Email: linescu@163.com

Abbreviations: BSAP, bone-specific alkaline phosphatase; CTX, C-terminal telopeptide of type I collagen; ELISA, enzyme-linked immunosorbent assay; NTX, N-terminal telopeptide of type I collagen; OC, osteocalcin; TRACP 5b, tartrate-resistant acid phosphatase 5b

Key words: nonunion, biomarkers, osteocalcin, tartrate-resistant acid phosphatase $5 \mathrm{~b}$, N-terminal telopeptide of type I collagen, C-terminal telopeptide of type I collagen, bone-specific alkaline phosphatase, enzyme-linked immunosorbent assay however, none of the bones had healed at 8 weeks. In the bone fracture group, the fracture line was distorted at 2 weeks and bone calluses formed at 6-8 weeks. In the bone defect group, the serum BSAP and TRACP 5b concentrations increased following the surgical procedure, peaked at 4 weeks, began to decrease at 5 weeks and stabilized after 6 weeks. The serum OC concentrations did not change significantly following the surgical procedure. The serum CTX concentrations fluctuated during the first 4 weeks, peaked at 5 weeks, then decreased and stabilized after 6 weeks. The serum NTX concentrations fluctuated during the first 4 weeks, were significantly lower at 5 weeks compared with the other time points and stabilized after 6 weeks. These results suggested that a bone nonunion model can be established in New Zealand rabbits by resecting a $15-\mathrm{mm}$ section of bone from the mid-radius prior to bone wax blocking. Measurement of the serum BSAP, CTX, NTX, and TRACP 5 b concentrations may be useful for the early detection of bone nonunion. The serum NTX concentrations changed significantly in rabbits with bone nonunion. Further studies are required in order to determine the feasibility of using serum NTX concentrations for the early diagnosis of bone nonunion.

\section{Introduction}

The diagnosis and treatment of bone nonunion has been extensively studied (1). The diagnosis and follow-up of nonunion rely predominantly on the interpretation of X-ray findings, which depend on the clinician's experience and the degree of bone callus mineralization during the fracture healing process (2). X-ray results are also affected by the projection, film processing conditions and subjective factors, resulting in poor accuracy (3). Furthermore, the X-ray resolution may be compromised when the bone mineral content is $<25 \%$ (4). Development of a novel and feasible method of monitoring bone healing is therefore urgently needed. B-mode ultrasound, response to vibration, mechanical impedance analysis and the creation of artificial neural networks have been extensively studied with respect to the function, mechanical status and changes of bone $(3,4)$. However, the feasibility of measuring serum biomarker concentrations for the monitoring of fracture healing remains unclear.

The present study investigated the diagnosis of fracture healing at the molecular level with the aim of developing a 
method for early diagnosis and outcome prediction of fracture nonunion, and of providing an objective method of assessing the efficacy of bone-growth drugs. As changes in bone metabolism precede changes in morphology, changes in biomarker levels can be detected earlier than changes in bone density and bone mass (2). However, the balance between bone formation and bone resorption in fracture nonunion remains unclear (5). The coupling of bone resorption and formation is closely associated with collagen metabolism, growth factors, vascular mediators and osteoblast activity (6). This study measured the serum concentrations of markers of bone turnover during the bone healing process. The serum biomarker concentrations were compared with the morphological changes on X-rays to determine whether they reflected bone nonunion, and to establish a database of serum biomarker concentrations. A rabbit model of bone nonunion was monitored by regular X-ray examinations. Serum biomarker concentrations were measured to investigate their usefulness for the early diagnosis of bone nonunion, and their changes in concentration during early bone nonunion.

\section{Materials and methods}

Reagents and apparatus. An ELISA kit for the determination of osteocalcin (OC; EIA-3375) was purchased from USCN Life Science, Inc. (Wuhan, China), whereas bone-specific alkaline phosphatase (BSAP; GCNML003), C-terminal telopeptide of type I collagen (CTX;XYI20748), N-terminal telopeptide of type I collagen (NTX;XYE10384) and tartrate-resistant acid phosphatase 5b (TRACP 5b; YZBIDSDO/2005) ELISA kits were purchased from Shanghai Langdun Shengwu (Shanghai, China). The present study used the following materials: Surgical instruments: Scalpel handle, scalpel blades, mosquito forceps, bone scalpel, rongeur, tissue scissors, suture scissors, sterile surgical towels, no. 1 silk sutures, operating table, bone wax and tape to secure the rabbits on the operating table; testing instruments: Vernier caliper (Ningbo Dahong Instrument, Ningbo, China) and X-ray machine (Shimadzu Corp., Kyoto, Japan); drugs: amoxicillin, 3\% pentobarbital sodium, ketamine, iodine (all Sanye Pharmaceutical Co., Ltd., Haikou, China) and lime (Tunchang Yinxin Lime Factory, Tunchang, China); and animal housing and feeding equipment: Rabbit cages and rabbit feed.

Animals and treatment. A total of 20 purebred New Zealand rabbits (10 male and 10 female; age, 5-6 months; weight, 2.5-3.0 kg) were purchased from the Medical Experimental Center of Hainan Provincial People's Hospital (Haikou, China) and maintained in separate cages for 12 weeks with natural light at $18-20^{\circ} \mathrm{C}$. Rabbits were fed on a diet of grass, hay, fresh vegetables, carrots, a limited amount of pellets three times a day and $300 \mathrm{ml}$ clean drinking water. Rabbits were randomly divided into two groups of 10 rabbits each: A bone defect group and a bone fracture group. In the bone defect group, a 15-mm section of bone (including the periosteum) was removed from the mid-radius, and the medullary cavities of the bone stumps were closed with bone wax. In the bone fracture group, the mid-radius was fractured, and no bone wax was used. No fracture fixation was performed in either group. Following the surgical procedure, the rabbits were housed in separate cages and allowed free activity. All animals received care in compliance with the 'Principles of laboratory animal care' (7).

Surgical methods. Rabbits were anesthetized with an intravenous injection of $3 \%$ pentobarbital sodium $(20 \mathrm{mg} / \mathrm{kg})$ via an ear vein and an intramuscular injection of $50 \mathrm{mg} / \mathrm{kg}$ ketamine. Once anesthetized, the rabbit was positioned on the operating table and secured with rope. The right forelimb was shaved and disinfected with iodine. A 2.5-3.0-cm skin incision was made over the mid-radius and the radius was exposed. In the bone defect group, a 15-mm length of bone was removed from the mid-radius. The Vernier caliper was used to ensure that the length of bone removed was accurate to within $0.1 \mathrm{~mm}$. The bone stumps were trimmed using rongeurs, and the defect was closed with bone wax. In the bone fracture group, the mid-radius was fractured. No fracture fixation was performed in either group. The skin wounds were closed using no. 1 silk sutures and were disinfected with iodine twice daily following the surgical procedure. The sutures were removed after 12 days. All rabbits were fed oral amoxicillin $(0.2-0.3 \mathrm{~g} / \mathrm{kg})$ twice daily for 1 week following the surgical procedure.

$X$-ray and biomarker examinations. X-rays were taken of the right forelimb of each rabbit preoperatively and at 2, 3, 4, 5, 6, $7,8,10$ and 12 weeks postoperatively to evaluate bone healing. A 2-ml blood sample was also taken at each time point and was stored at $-80^{\circ} \mathrm{C}$ for measurement of serum biomarker concentrations.

ELISA determination of biomarker concentrations. The serum biomarker concentrations measured were those of OC and BSAP, which served as markers of bone formation, and those of CTX, NTX and TRACP 5b, which served as markers of bone resorption.

All biomarker concentrations were measured by biotin double-antibody sandwich ELISA, according to the manufacturers' protocols. For each concentration determination, the ELISA plate was embedded with the respective monoclonal antibody and the biomarker was subsequently added. After the monoclonal antibody had been incubated with the biomarker, a biotin-labeled antibody against the biomarker was added to the mixture, thus binding to streptavidin-horseradish peroxidase and forming immune complexes. The complexes were subsequently incubated and rinsed with the wash solution provided in the kit to eliminate unbound enzyme, developed in a blue stain with chromogen $\mathrm{A}$ and B, and finally developed in a yellow stain with the acid. The degree of staining was positively correlated with the concentration of the biomarker in the specimen.

Statistical analysis of serum biomarker concentrations. All data are expressed as means \pm standard deviation and were analyzed using SPSS 20.0 software (SPSS, Inc., Chicago, IL, USA). One-way analysis of variance was used to estimate average values, followed by effect comparisons between groups, a spherical test, intragroup (repeated) effect comparisons, and interaction effect comparisons. $\mathrm{P}<0.05$ was considered to indicate a statistically significant difference. Sample sizes were determined based on a preselected power level of 0.8 . 
A

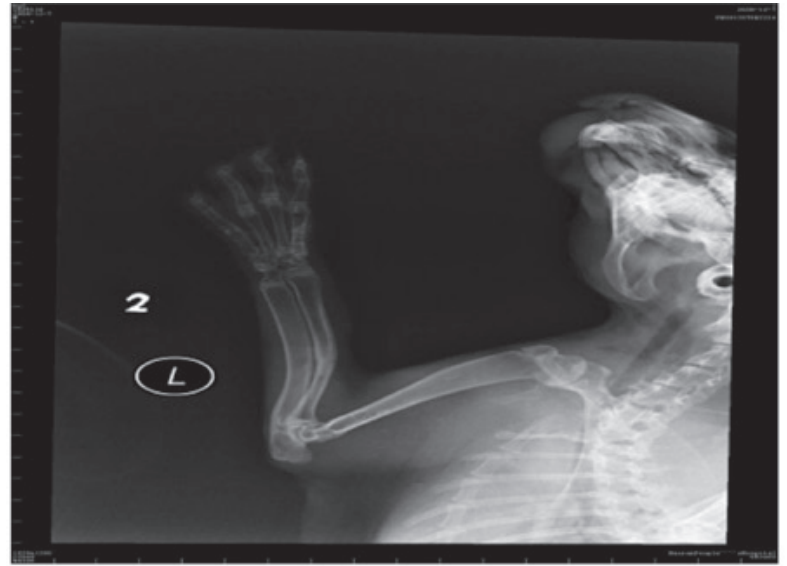

B

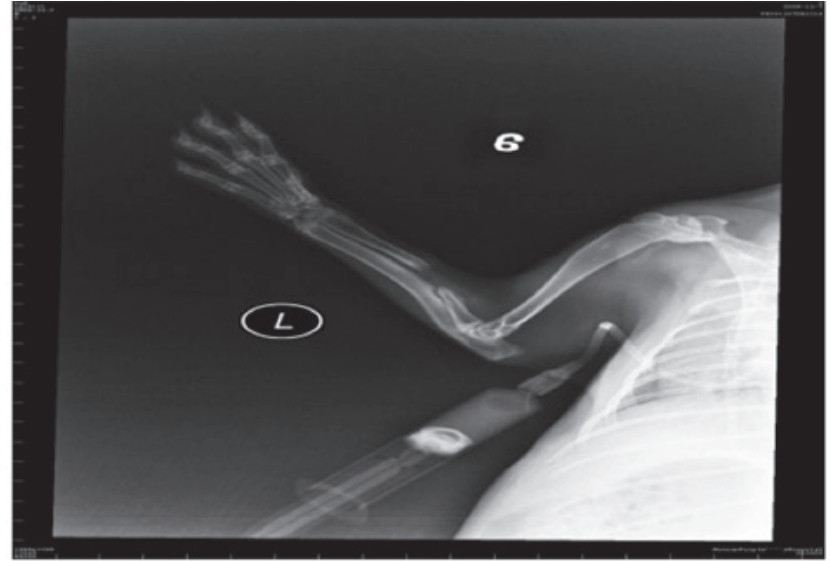

Figure 1. Eight weeks postoperative X-ray observation demonstrated that a large number of bone calluses had formed in the (A) bone fracture group and none of the bones had healed in the (B) bone defect group.

\section{Results}

Observation of the rabbits following the surgical procedure. None of the rabbits developed infection of the surgical incision, and none died during the experimental period. All the rabbits were included in all the analyses.

$X$-ray findings. In the bone defect group, bone callus formation was observed in three rabbits at 2 weeks and the bone calluses stabilized at 5 weeks, but none of the bones had healed at 8 weeks (Fig. 1A). In the bone fracture group, the fracture line was distorted at 2 weeks and a large number of bone calluses formed at 6-8 weeks (Fig. 1B).

Serum biomarker concentrations. Serum BSAP (Fig. 2), CTX (Fig. 3), OC (Fig. 4) and NTX (Fig. 5) concentrations were significantly different between the two groups (OC: $\mathrm{F}=22.989$, $\mathrm{P}<0.001$; BSAP: $\mathrm{F}=16.051, \mathrm{P}=0.001$; CTX: $\mathrm{F}=27.737, \mathrm{P}<0.001$; NTX: $F=187.512, P<0.001)$. There were no significant differences in the serum OC, BSAP, CTX or TRACP $5 b$ (Fig. 6) concentrations within each group at the various time points. In the bone defect group, serum BSAP concentration increased postoperatively and peaked at 4 weeks, began to decrease at 5 weeks and stabilized after 6 weeks (Fig. 2). Serum CTX concentrations fluctuated during the first 4 weeks, peaked at 5 weeks, then decreased and stabilized after 6 weeks (Fig. 3). Serum OC concentrations did not significantly alter following the surgical procedure (Fig. 4). In the bone defect group, the serum NTX concentrations were significantly lower at 5 weeks compared with the other time points prior to and following the surgical procedure (Fig. 5). There were significant differences between the serum NTX concentrations at 7, 8, 10, and 12 weeks and at 3, 4 and 5 weeks. The serum NTX concentrations stabilized following 6 weeks. Serum TRACP $5 b$ concentration increased following the surgical procedure and peaked at 4 weeks, then decreased and remained low (Fig. 6).

\section{Discussion}

In recent years, bone defects caused by nonunion, delayed healing, and other factors have attracted increasing attention, and have been studied in animal experiments (8). Animals
BSAP (nmol/l)

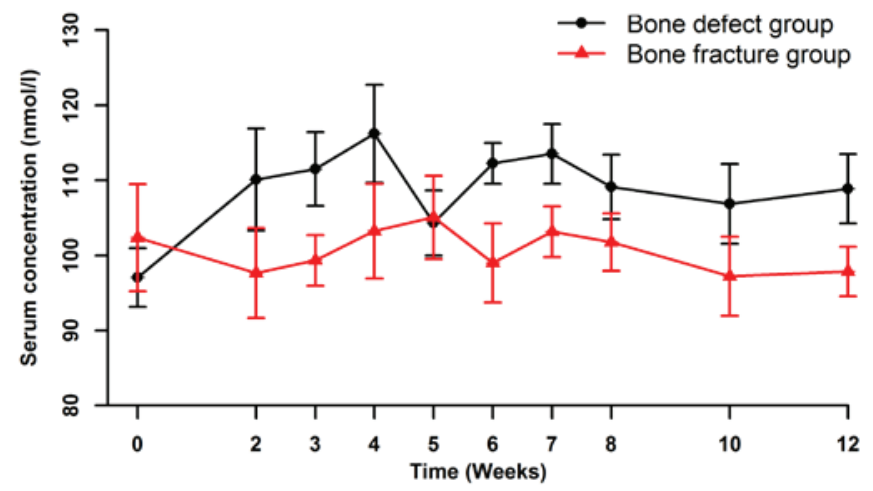

Figure 2. Serum concentrations of BSAP at various time points, including prior to the surgical procedure and up to 12 weeks after surgery. Data are presented as the mean \pm standard deviation. BSAP, bone-specific alkaline phosphatase.

\section{$\operatorname{CTX}(\mathrm{nmol} / \mathrm{l})$}

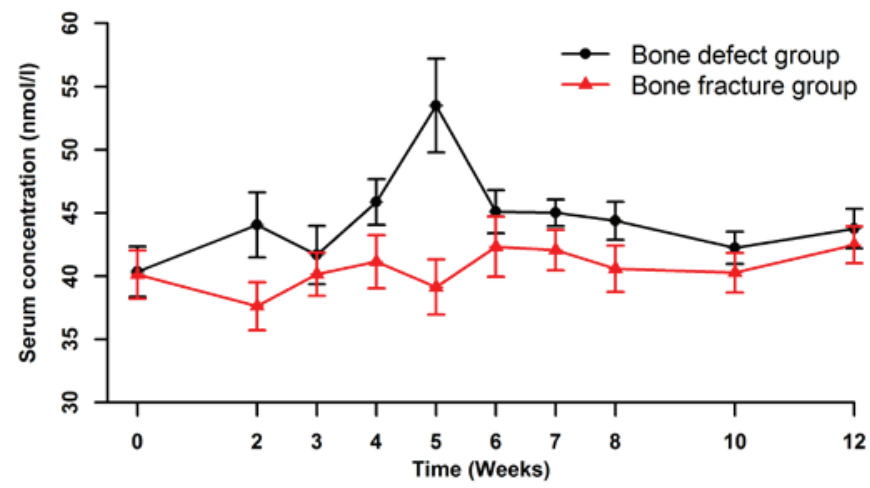

Figure 3. Serum concentrations of CTX at various time points, including prior to the surgical procedure and up to 12 weeks after surgery. Data are presented as the mean \pm standard deviation. CTX, C-terminal telopeptide of type I collagen.

have anatomical and physiological characteristics similar to those of humans, and animal experiments can be used to model human conditions such as bone nonunion with good reproducibility. Experimental studies of bone nonunion have 


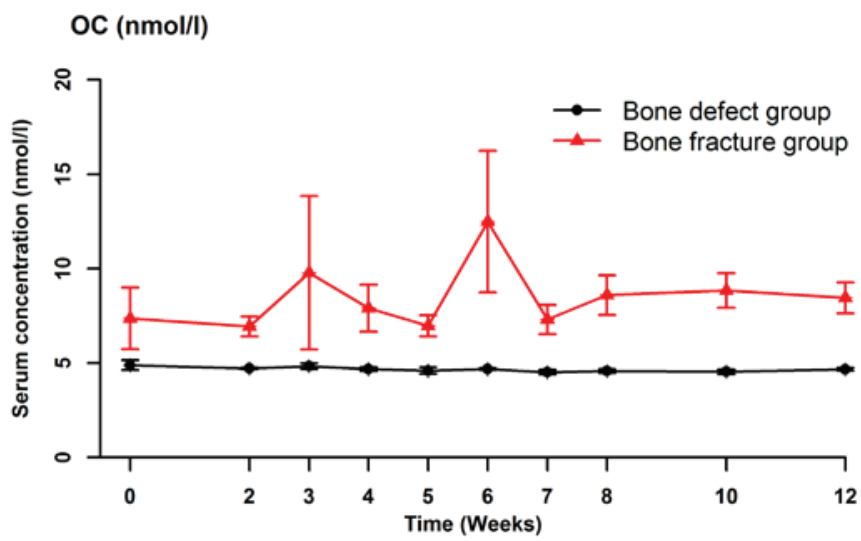

Figure 4. Serum concentrations of OC at various time points, including prior to the surgical procedure and up to 12 weeks after surgery. Data are presented as the mean \pm standard deviation. OC, osteocalcin.

\section{NTX (nmol/l)}

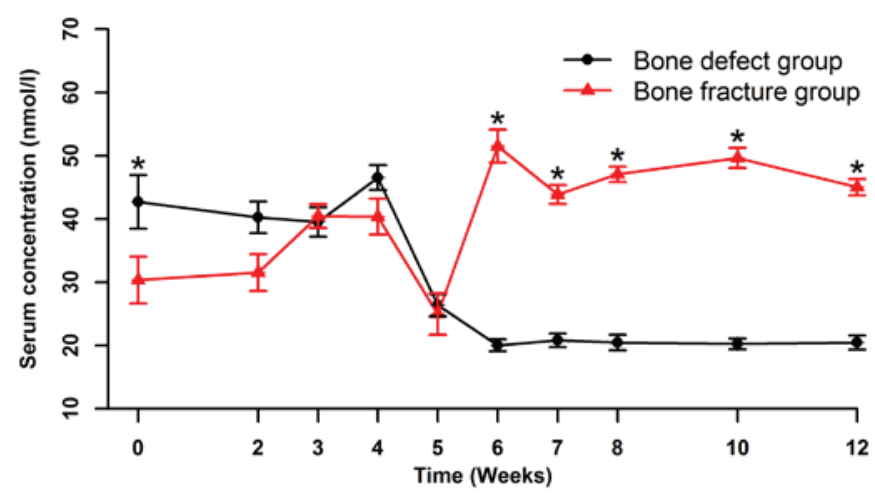

Figure 5. Serum concentrations of NTX at various time points, including prior to the surgical procedure and up to 12 weeks after surgery. Data are presented as the mean \pm standard deviation. NTX, N-terminal telopeptide of type I collagen.

focused on large animals such as pigs, horses, cows, sheep and monkeys $(9,10)$. However, use of these large animals has some disadvantages, such as high cost, demanding feeding conditions, and difficult experimental designs (11). Although small animals are not suitable for models of fracture fixation, they recover rapidly from trauma and are suitable for cytological studies of fracture healing (6). A previous study reported that rabbits are ideal for cytological studies of fracture healing due to the similarities between rabbit and human limbs, the small body size, ease of handling, rapid tissue repair, short breeding cycle, simple breeding management, low cost, and availability of large sample sizes (12). New Zealand white rabbits were therefore chosen for the present study.

Establishment of useful animal models of bone defects is necessary for tissue engineering research of bone nonunion. The length of the bone defect should be sufficient to prevent union of the bone. Bone healing depends predominantly on the size of the defect, but is also influenced by the age, body mass and sex of the animal (13). The forelegs of New Zealand white rabbits have a radius and an ulna. When a defect is created in the mid-radius, the ulna continues to support the forelimb (9). As internal fixation is not needed, this is a
TRACP5b (nmol/l)

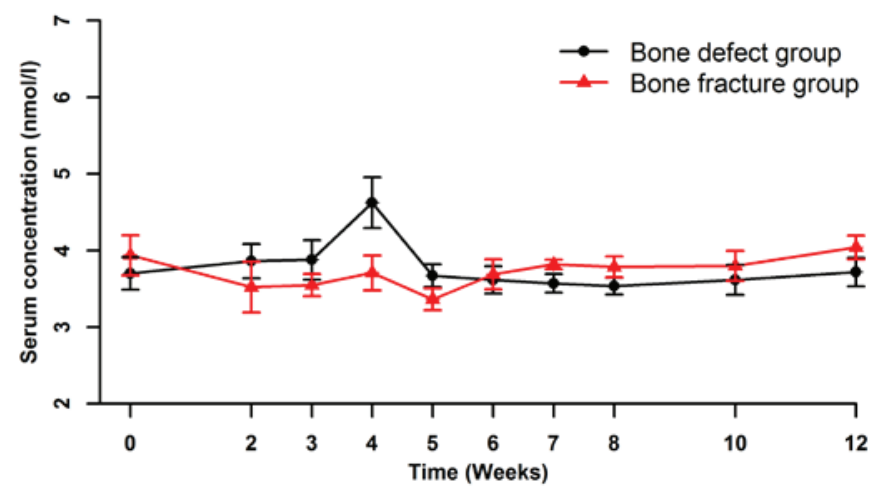

Figure 6. Serum concentrations of TRACP $5 \mathrm{~b}$ at at various time points, including prior to the surgical procedure and up to 12 weeks after surgery. The data points show the mean absolute values. TRACP 5b, tartrate-resistant acid phosphatase $5 \mathrm{~b}$.

commonly used model. Johnson et al (14) demonstrated that certain bone defects are not conducive to bone growth and repair, such as a defect length of >3-4 times the diameter of the bone shaft in an adult animal, a defect covered by local muscle tissue, or a defect in a bone with low red bone marrow content. The optimal length of an experimental defect in the radius is controversial. According to the Stephen standard, the defect should have a length of 1.5-2.0 times the diameter of the bone $(13,15)$. As the diameter of the radius in New Zealand rabbits is 4.0-5.0 mm, many investigators use 15-mm long mid-radius bone defects. Kasten et al (16), Niemeyer et al (17) and other researchers (18-21) have demonstrated that the area of the bone defect was filled with fibrous scar tissue without a bony connection, the bone wax was not absorbed, the bone stumps were hardened, the medullary cavity was blocked, and a small bone callus had formed 10 weeks following the creation of a $15-\mathrm{mm}$ long defect in the radius of a rabbit.

In the present study, the results of the X-ray examinations indicated that the $15-\mathrm{mm}$ bone defects were connected by fibrous tissue and that the medullary cavity at the bone stumps was blocked at 12 weeks postoperatively. The fracture healing time in rabbits is usually $\sim 6$ weeks, and absence of healing after 12 weeks indicates nonunion. The $15-\mathrm{mm}$ bone defects in the rabbits of the present study had not healed after 12 weeks, indicating successful establishment of a bone nonunion model.

The existing methods used to study nonunion include measurements of bone morphometry, bone mineral density, and the bone metabolic index (22). The bone metabolic index is of particular interest because it can be measured non-invasively and repeatedly (23). Bone formation, bone resorption, and static conditions contribute to the process of bone reconstruction (24). Bone turnover involves the continuous removal of old bone by osteoclasts and simultaneous formation of osteoid and mineralization by osteoblasts. These two processes are tightly coupled. The bone resorption-remodeling process is regulated by osteoblasts (25). The bone mass is determined by the balance of bone formation and bone resorption in the same bone reconstruction unit (17). When this balance is disrupted and the rate of bone resorption is 
higher than the rate of bone formation, nonunion occurs (26). Serum biomarker concentrations can reflect the status of the bone turnover process by indirectly measuring osteoblast and osteoclast activity (27).

A number of sensitive and specific biomarkers have been studied for the monitoring of bone loss, prediction of fracture risk, evaluation of drug treatment responses, and differential diagnosis of metabolic bone diseases (28). The main biomarkers are BSAP, CTX, NTX, and TRACP 5b $(23,25)$. Measurement of the serum concentrations of these biomarkers enables the earlier assessment of treatment effects than measurement of bone density.

BSAP is an extracellular enzyme produced by osteoblasts that is not affected by diseases of the liver, kidney, or intestines (29). The serum BSAP concentration is a specific and sensitive indicator of osteoblast activity and bone formation, and a high concentration indicates a high level of osteoblast activity (27). Previous studies have reported that the serum BSAP concentration reflects bone turnover $(27,28)$. Southwood et al (30) studied a rabbit model of femur defects, and found that the serum BSAP concentration was low during the first 4 weeks after the surgical procedure, increased to a peak at 8 weeks, and then decreased. Moghaddam et al (31) compared 15 patients with atrophic nonunion following a long bone shaft fracture with 15 matched patients with normal fracture healing selected from a pool of 248 patients who underwent orthopedic surgery. They measured serum biomarker concentrations at 1, 2, 4, 8, 12 and 52 weeks after surgery, and demonstrated that the serum BSAP and procollagen type I N-terminal propeptide concentrations initially increased and then decreased during the first week following the surgical procedure. There were no significant differences in the absolute or relative concentrations of these biomarkers between the two groups during the healing process. In the bone defect group in the present study, the serum BSAP concentration increased postoperatively and peaked at 4 weeks, then began to decrease at 5 weeks and stabilized after 6 weeks. The serum BSAP concentrations were significantly different between the two groups, but were not significantly different between the various time points within each group. These results indicated that the bone defect stimulated bone formation resulting in an increase in serum BSAP concentrations, which was similar to the results obtained by Southwood et al (30) and Moghaddam et al (31). Changes in the serum BSAP concentration can be used to monitor bone healing in experimental rabbits.

In both animals and humans, $\mathrm{OC}$ is produced and secreted by non-proliferating osteoblasts (32). OC is the main component of non-collagenous proteins in the bone tissue, and contains 49 amino acids and 3 carboxyglutamic acid residues (33). The biological activity of OC depends on vitamins $\mathrm{K}$ and $\mathrm{D}$. Mature OC is predominantly located in bone tissue outside the mesenchymal cells and dentin, and a small amount is released into the blood (33). In 1988, Eastell et al (34) proposed that the serum OC concentration is a sensitive and specific biomarker of bone turnover and bone formation. OC is currently a major focus of studies of bone metabolism. In vitro and in vivo experiments have demonstrated that $\mathrm{OC}$ is associated with the regulation of bone resorption as well as in osteoblast differentiation and matrix mineralization (33-35). Delmas et al (36) demonstrated that serum OC concentrations reflect bone turnover when bone formation is coupled with bone resorption, but reflects only bone formation when bone formation is not coupled with bone resorption. Previous Chinese studies have reported that a high serum OC concentration was associated with high bone mineral density, suggesting that bone formation and bone mass will increase when the serum OC concentration is high $(31,32)$. The serum OC concentration therefore reflects bone mineral density and can be used to predict bone nonunion. In the present study, the serum OC concentration did not change following the surgical procedure in the bone defect group, in spite of changes in the bone mineral density. This indicates that the serum OC concentration is not a sensitive indicator of bone nonunion in rabbits.

TRACP is one of six acid phosphatase isozymes and is predominantly secreted by osteoclasts (37). The serum TRACP concentration may reflect osteoclast activity and bone resorption in vivo (38). Under normal conditions, two types of TRACP 5 are present in human serum: $5 \mathrm{a}$ and 5b (39). Sialidase converts TRACP 5a to TRACP 5b, which is the purified human osteoclast TRACP. The serum TRACP 5b concentration is regarded as the optimal biomarker of bone resorption due to its high specificity, lack of diurnal variation, and independence of diet and liver or kidney diseases. Measurement of the serum TRACP 5b concentration can also be used for the early detection of osteoporosis, thereby reducing the risk of fractures (40).

CTX contains important intermolecular cross-linking agents of type I collagen and residues of cross-linking agents, and is not degraded by the kidney (39). The serum CTX concentration is therefore an ideal marker of bone resorption. CTX has three different forms: CTX-matrix metalloproteinase, $\alpha-\mathrm{CTX}$, and $\beta$-CTX, which are collectively termed CrossLaps. $\alpha$-CTX and $\beta$-CTX both have only eight amino acid sequences $(40,41)$.

Moghaddam et al (31) measured serum biomarker concentrations in 15 patients with atrophic nonunion with 15 matched patients with normal fracture healing selected from a pool of 248 patients who underwent orthopedic surgery, as described above. At 1 week after surgery, the serum CTX concentration was significantly lower in the nonunion group compared with the normal healing group. At 4 and 8 weeks, the serum TRACP 5b concentration was significantly lower in the nonunion group. The absolute and relative serum TRACP 5 b concentrations were not significantly different between the two groups at any of the time points. In the bone defect group in the present study, the serum TRACP $5 \mathrm{~b}$ concentration increased following the surgical procedure and peaked at 4 weeks, then decreased and remained low. The serum CTX concentrations changed in a similar pattern in the bone defect and bone fracture groups, but with significant differences between the two groups. The serum CTX concentrations peaked at 5 weeks following the surgical procedure in the bone defect group, but there were no significant differences in CTX concentration between the various time points within each group. The serum TRACP 5b and CTX concentrations increased following surgery in the bone defect group, indicating increased bone resorption. The serum TRACP 5b and CTX concentrations may reflect the early healing process, and may be useful for clinical monitoring. 
NTX is a low-molecular-weight peptide that contains hydroxylysyl pyridinoline and lysyl pyridinoline, one of which is connected to each end of the peptide chain (42). NTX is a type I collagen cross-linked telopeptide and the total $\mathrm{N}$-terminal cross-linking agent (41-43). Lysyl pyridinoline is predominantly located within the bone and accounts for $21 \%$ of mature collagen, and hydroxylysyl pyridinoline is located in both cartilage and bone and is a major component of mature collagen (36). Lysyl pyridinoline and hydroxylysyl pyridinoline are released from the bone matrix in the process of osteoclastic bone resorption, circulate as free amino acids or bound to peptides such as NTX, and are excreted in the urine $(38,44)$. NTX is a stable peptide excreted in the urine following bone resorption; therefore a high serum NTX concentration is likely highly specific for bone resorption (31). At the beginning of 1990s, Hanson et al (45) established an ELISA method to determine NTX concentration, following which the investigators determined that there were immunologically competent NTX in the culture medium when osteoclasts were cultured in vitro. García-Pérez et al (26) reported that the serum NTX concentration was a sensitive indicator of bone resorption. Rosen et al (46) demonstrated that the serum NTX concentration was negatively correlated with the bone mineral density, and that a higher baseline serum NTX concentration indicated a more rapid decline in bone density. In the bone defect group in the present study, the serum NTX concentration was significantly lower at 5 weeks following the surgical procedure than prior to surgery and stabilized after 6 weeks. The serum NTX concentrations were significantly different between the bone defect and bone fracture groups.

In the present study, the serum OC concentration did not change significantly following the surgical procedure in the bone defect group, whereas the serum BSAP, CTX, and TRACP 5b concentrations fluctuated postoperatively. Although the serum OC, BSAP, CTX, and TRACP 5b concentrations did not change significantly following surgery in the bone defect group, this group had a high bone turnover rate. Measurement of serum biomarker concentrations may help with clinical evaluation of the early healing process. In the bone defect group, the serum NTX concentration was significantly lower at 5 weeks following the surgical procedure than before surgery, suggesting that the serum NTX concentration provides an accurate reflection of bone turnover in vivo and changes quickly following changes in bone resorption. Further studies are required in order to definitively determine whether the serum NTX concentration is a sensitive and specific indicator of bone resorption, and whether the serum NTX concentration accurately reflects early bone turnover and can predict bone nonunion.

\section{Acknowledgements}

The present study was supported by a grant from the Natural Science Foundation of Hainan Province, China (grant no. 808212).

\section{References}

1. Court-Brown CM and McQueen MM: Nonunions of the proximal humerus: their prevalence and functional outcome. J Trauma 64: 1517-1521, 2008.
2. Blokhuis TJ, de Bruine JH, Bramer JA, den Boer FC, Bakker FC, Patka P, Haarman HJ and Manoliu RA: The reliability of plain radiography in experimental fracture healing. Skeletal Radio 30: 151-156, 2001

3. Lotz J, Gaertner T, Hahn M and Prellwitz W: Collagen type I metabolism after bone surgery. Arch Orthop Trauma Surg 119: 212-216, 1999.

4. Sambrook P and Cooper C: Osteoporosis. Lancet 367: 2010-2018, 2006.

5. Lin JP, Song SF and Yao LL: Research progress of fracture healing and it's early diagnosis. Ortho J China 24: 1876-1878, 2009.

6. Gamero P and Somay-Rendu E: Biochemical markers of bone loss rate and prevalence of osteoporosis at multiple skeletal sites in Chinese women. Osteoporos Int 13: 13: 669-676, 2002.

7. Laboratory animal welfare: Public Health Service policy on humane care and use of laboratory animals by awardee institutions; notice. Fed Regist 50: 19584-19585, 1985.

8. Kokubu T, Hak DJ, Hazelwood SJ and Reddi AH: Development of an atrophic nonunion model and comparison to a closed healing fracture in rat femur. J Orthop Res 21: 503-510, 2003.

9. Reed AA, Joyner CJ, Brownlow HC and Simpson AH: Human atrophic fracture nonunion are not avascular. J Orthop Res 20: 593-599, 2002.

10. Mumaghan M, Li G and Mash DR: Nonsteroidal antiinflammatory drug induced fracture nonunion: An inhibition of angiognensis? JBJS Am 88: 141-147, 2006.

11. Nunamaker DM: Experimental models of fracture repair. Clin Orthop (Suppl 355): 56, 1998.

12. Peter CP and Cook WO: Effect of alendronate on fracture healing and bone remodeling in dogs. J Orthop Res 14: 74, 1996.

13. Korkmaz M, Oztürk H, Bulut O, Unsaldi T and Kaloğlu C: The effect of definitive continuous distraction employed with the Ilizarov type external fixation system on fracture healing: An experimental rabbit model. Acta Orthop Traumatol Turc 39: 247-257, 2005 (In Turkish).

14. Johnson EE, Urist MR, Schmalzried TP, Chotivichit A, Huang HK and Finerman GA: Autogeneic cancellous bone grafts in extensive segmental ulnar defects in dogs. Effects of xenogeneic bovine bone morphogenetic protein without and with interposition of soft tissues and interruption of blood supply. Clin Orthop Relat Res: 254-265, 1989.

15. Cook SD, Wolfe MW, Salkeld SL and Rueger DC: Effect of recombinant human osteogenic protein-1 on healing of segmental defects in non-human primate. J Bone Joint Surg Am 77: 734-750, 1995.

16. Kasten P, Vogel J, Geiger F, Niemeyer P, Luginbühl R and Szalay K: The effect of platelet-rich plasma on healing in critical-size long-bone defects. Biomaterials 29: 3983-3992, 2008.

17. Niemeyer P, Szalay K, Luginbühl R, Südkamp NP and Kasten P: Transplantation of human mesenchymal stem cells in a non-autogenous setting for bone regeneration in a rabbit critical-size defect model. Acta Biomater 6: 900-908, 2010.

18. Felix R, Herrmann W and Fleisch H: Stimulation of precipitation of calcium phosphate by matrix vesicles. Biochem J 170: 681-691, 1978.

19. Anderson HC, Stechschulte DJ Jr, Collins DE, Jacobs DH, Morris DC, Hsu HH, Redford PA and Zeiger S: Matrix vesicle biogenesis in vitro by rachitic and normal rat chondrocytes. Am J Pathol 136: 391-398, 1990.

20. Parker MJ, Raghavan R and Gurusamy K: Incidence of fracture-healing complications after femoral neck fractures. Clin Orthop Relat Res 458: 175-179, 2007.

21. Gothlin G and Ericsson JL: Fine structural localization of alkaline phosphatase in the fracture callus of the rat. Histochemie 36: 225-236, 1973.

22. Pedersen BJ, Schlemmer A, Hassager C and Christiansen C: Changes in the carboxyl-terminal propeptide of type I procollagen and other markers of bone formation upon five days of bed rest. Bone 17: 91-95, 1995.

23. Lian J, Stewart C, Puchacz E, Mackowiak S, Shalhoub V, Collart D, Zambetti G and Stein G: Structure of the rat osteocalcin gene and regulation of vitamin D-dependent expression. Proc Natl Acad Sci USA 86: 1143-1147, 1989.

24. Noite PA, Vander Krans A, Patka P, Janssen IM, Ryaby JP, Albers GH: Low-intensity pulsed ultrasound device for the noninvasive treatment of nonunions. J Trauma 51: 693-702, 2001. 
25. Geiger F, Lorenz H, Xu W, Szalay K, Kasten P, Claes L, Augat P and Richter W: VEGF producing bone marrow stromal cells (BMSC) enhance vascularization and resorption of a natural coral bone substitute. Bone 41: 516-522, 2007.

26. García-Pérez MA, Moreno-Mercer J, Tarín JJ and Cano A: Similar efficacy of low and standard doses of transdermal estradiol in controlling bone turnover in postmenopausal women. Gynecol Endocrinol 22: 179-184, 2006

27. Kyro A, Usenius JP, Aarnio M, Kunnamo I and Avikainen V: Are smokers a risk group for delayed healing of tibial shaft fractures? Ann Chir Gynaecol 82: 254-262, 1993.

28. Adams CI, Keating JF and Court-Brown CM: Cigarette smoking and open tibial fractures. Injury 32: 61-65, 2001

29. Kurdy NM: Serology of abnormal fracture healing: The role of PINP, PICP, and BsALP. J Orthop Trauma 14: 48-53, 2000

30. Southwood LL, Frisbie DD, Kawcak CE and McIlwraith CW: Evaluation of serum biochemical markers of bone metabolism for early diagnosis of nonunion and infected nonunion fractures in rabbits. Am J Vet Res 64: 727-735, 2003.

31. Moghaddam A, Müller U, Roth HJ, Wentzensen A, Grützner PA and Zimmermann G: TRACP 5b and CTX as osteological markers of delayed fracture healing. Injury 42: 758-764, 2011.

32. Kaplan FS, Hayes WC, Keaveny TM, Boskey AL, Einhorn TA and Iannotti JP: Form and function of bone. In: Orthopaedic basic science. Simon SS (ed). Amercan Academy of Orthopaedic Surgeons, Rosemont, IL, pp127-184, 1994.

33. Linkhart SG, Linkhart TA, Taylor AK, Wergedal JE, Bettica P and Baylink DJ: Synthetic peptidebased immunoassay for amino-terminal propeptide of type I procollagen: application for evaluation of bone formation. Clin Chem 39: 2254-2258, 1993.

34. Eastell R, Yergey AL, Vieira NE, Cedel SL, Kumar R and Riggs BL: Interrelationship among vitamin D metabolism, true calcium absorption, parathyroid function, and age in women: Evidence of an age-related intestinal resistance to 1,25-dihydroxyvitamin D action. J Bone Miner Res 6: 125-132, 1991

35. Sato Y, Kaji M, Higuchi F, Yanagida I, Oishi K and Oizumi K: Changes in bone and calcium metabolism following hip fracture in elderly patients. Osteoporos Int 12: 445-449, 2001.
36. Delmas PD: Biochemical markers of bone turnover for the clinical assessment of metabolic bone disease. Endocrinol Metab Clin North Am 19: 1-18, 1990.

37. Seibel MJ: Molecular markers of bone turnover:Biochemical technical and analytical aspects. Osteoporos Int 11: 18-29, 2000.

38. Lin JP, Song SF and Yao LL: Feasibility of predicting fracture risk with bone turnover markers and bone mineral density. Zhongguo Zuzhi Gongcheng Yanjiu yu Linchuang Kangfu 14:317-320, 2010.

39. Szulc P and Delmas PD: Biochemical markers of bone turnover in men. Calcif Tissue Int 69: 229-234, 2001.

40. Laurer HL, Hagenbourger O, Quast S, Herrmann W and Marzi I: Sequential changes and pattern of bone specific alkaline phosphtase after trauma. Eur J Trauma 1: 33-38, 2000.

41. Dahabreh Z, Calori GM, Kanakaris NK, Nikolaou VS and Giannoudis PV: A cost analysis of treatment of tibial fracture nonunion by bone grafting or bone morphogenetic protein-7. Int Orthop 33: 1407-1414, 2009.

42. Borys J, Grabowska SZ, Antonowicz B, Dryl D, Citko A and Rogowski F: The concentuation of C-terminal propeptide of type I procollagen in blood serum in the course of mandibular fracture healing(preliminary report). Rocz Akad Med Bialmyst 46: 251-262, 2001.

43. Ingle BM, Hay SM, Bottjer HM and Eastell R: Changes in bone mass and bone turnover following ankle fracture. Osteporos Int 10: 408-415, 1999

44. Kon T, Cho TJ, Aizawa T, Yamazaki M, Nooh N, Graves D, Gerstenfeld LC and Einhorn TA: Expression of osteoprotegerin, receptor activator of NF-kappaB ligand (osteoprotegerin ligand) and related proinflammatory cytokines during fracture healing. J Bone Miner Res 16: 1004-1014, 2001.

45. Hanson DA, Weis MA, Bollen AM, Maslan SL, Singer FR and Eyre DR: A specific immunoassay for monitoring human bone resorption: Quantitation of type I collagen cross-linked N-telopeptides in urine. J Bone Miner Res 7: 1251-1258, 1992.

46. Rosen HN, Moses AC, Garber J, Ross DS, Lee SL and Greenspan SL: Utility of biochemical markers of bone turnover in the follow-up of patients treatment with biphosphonates. Calcif Tissue Int 63: 363-368, 1998. 